\title{
DEVELOPMENT OF THE SIGNAL ARRANGEMENT EVALUATION SYSTEM
}

\author{
KAZUMASA KUMAZAWA, TAKAYASU KITANO, TAKASHI SAKAGUCHI, \\ SHUNICHI TANAKA \& SATOSHI KATO \\ Signalling and Transport Information Technology Division, Railway Technical Research Institute, Japan
}

\begin{abstract}
In order to construct new lines or to change track layouts in stations, it is necessary to determine the position of each signal, called an examination of the signal arrangement, in consideration of train operation and so on. To obtain the signal arrangement that satisfies the desired train operation, it is essential to consider headways. Furthermore, in the automatic block section, the signal aspect transition between adjacent signals must be determined, taking account of the position of each signal and so on. Therefore, to determine a signal arrangement requires a lot of time and a person who has the knowledge of both signalling system and train operation. To solve these problems, we developed a signal arrangement evaluation system. The system has two main functions. One function is the creation of the signal aspect transitions. In this function, this system calculates a braking distance corresponding to a signal aspect transition and checks whether the braking distance is lower than a distance between two signals or not. The other function is the creation of a headway diagram. In this function, the system calculates headways and checks whether the headways of all signals satisfy the desired train operation or not. In this paper, we explain these calculation logics and the structure of the developed system. In addition, we introduce a result of evaluating a signal arrangement in a model line by using the developed system.
\end{abstract}

Keywords: signalling system, train performance curve, train headway, automatic block system, signal aspect, examination of new railway lines, track layouts improvement.

\section{INTRODUCTION}

When planning new line construction and track layouts improvement, running time between stations and time interval between trains are important. Running time can be calculated from the characteristics of the rolling stock and infrastructure conditions such as gradients of a track, speed limits and the position of station. In addition, a signal arrangement is required to calculate time interval between trains. The task of deciding signal positions based on a target time interval is called an examination of a signal arrangement. The examination of the signal arrangement consists of four tasks. First, a signal aspect transitions that represents transitions between signal aspects is created based on the signal arrangement plan. Secondly, the train headway (hereinafter called the headway) that indicates time interval between trains is calculated based on the signal aspect transitions. Thirdly, headways at each signal are conformed whether it satisfies the target value or not. Finally, the above tasks are repeated until the target value of the headway is satisfied. Furthermore, in creating the signal aspect transitions, it is necessary to perform deceleration distance calculation for all transitions between signal aspects, and repeat the adjustment of the signal arrangement plan based on the result. The examination of the signal arrangement requires a lot of time and a skilled person who has the knowledge of signalling system and train operation. For that reason, system assistance is required to carry out the examination of the signal arrangement.

In order to solve these problems, we developed a signal arrangement evaluation system. First, we conducted hearing investigations with the person in charge of the examination of the signal arrangement to understand the overview of it. Based on the knowledge we have obtained from these investigations, we developed a system that can evaluate the signal 
transitions and the headway at each signal. Then, we applied the developed system to a model line and confirmed its usefulness.

\section{OVERVIEW OF AN EXAMINATION OF THE SIGNAL ARRANGEMENT}

As shown in Fig. 1, the general procedure for an examination of the signal arrangement on several Japanese suburban lines is as follows: Step 1: creating a signal arrangement plan according to the examination conditions. Step 2: creating signal aspect transitions based on the signal arrangement plan. Step 3: calculation headways at each signals based on the signal aspect transitions and train performance curves, and Step 4: evaluating the signal arrangement. In the examination of the signal arrangement, the above Steps 1 to 4 are repeated to determine signal arrangement in which $t$ the value of headways at all signals satisfies that of the target headway.

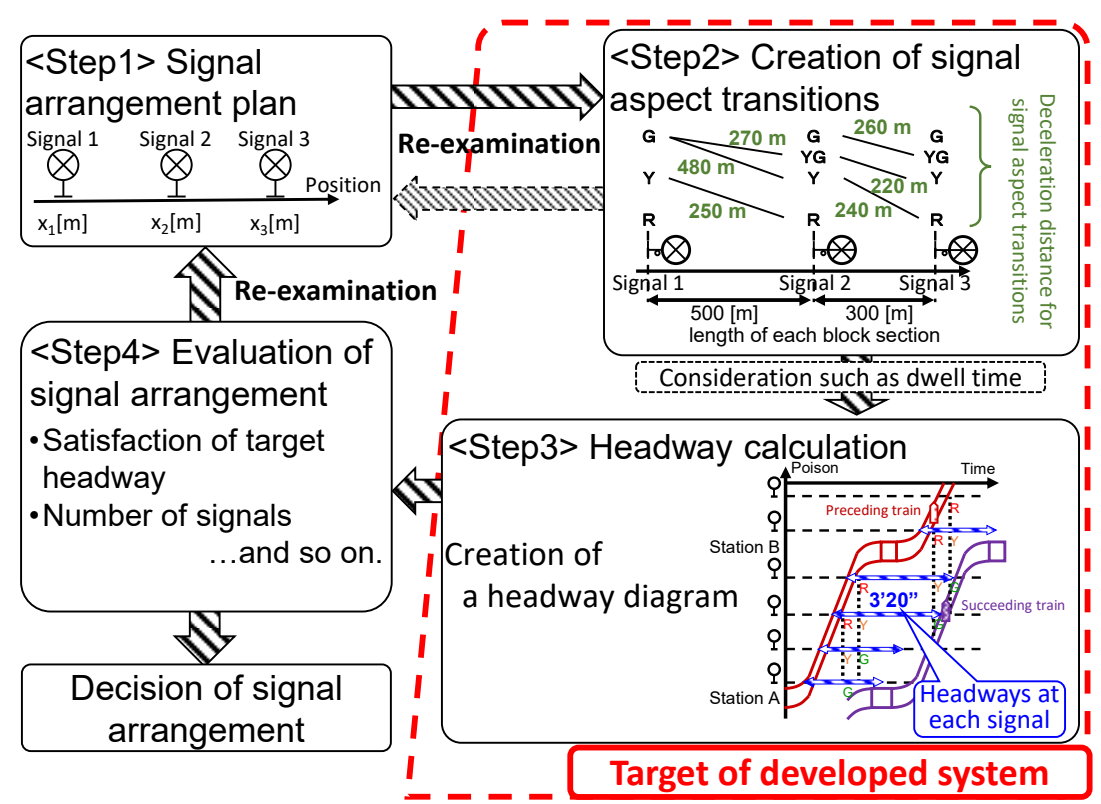

Figure 1: Overview of an examination of the signal arrangement.

In this paper, we focus on the creation of the signal aspect transitions (Step 2) and the calculation of headways (Step 3), which are important and time-consuming tasks in the examination of the signal arrangement. In order to develop the signal arrangement evaluation system, it is necessary to conduct hearing investigations with the person in charge of the examination of the signal arrangement and obtain their knowledge. This section summarizes the knowledge obtained from the investigation on Step 2 and Step 3.

\subsection{Target signalling system}

We focus on the Japanese automatic block system. In the automatic block section, main signals divide the line into several sections, and each section is set as a block [1]. Interval between trains is controlled by the signal aspect of the main signal. The signal aspect has the 
Table 1: An example of directed speed by a signal [2].

\begin{tabular}{llc}
\hline Signal indication & Signal aspect & Directed speed \\
\hline \hline Proceed signal & Green $(\mathrm{G})$ & Line speed \\
\hline Reduced speed signal & Yellow and Green $(\mathrm{YG})$ & $65 \mathrm{~km} / \mathrm{h}$ \\
\hline Caution signal & Yellow $(\mathrm{Y})$ & $45 \mathrm{~km} / \mathrm{h}$ \\
\hline Restricted speed signal & Two Yellows (YY) & $25 \mathrm{~km} / \mathrm{h}$ \\
\hline Stop signal & Red $(\mathrm{R})$ & $0 \mathrm{~km} / \mathrm{h}$ \\
\hline
\end{tabular}

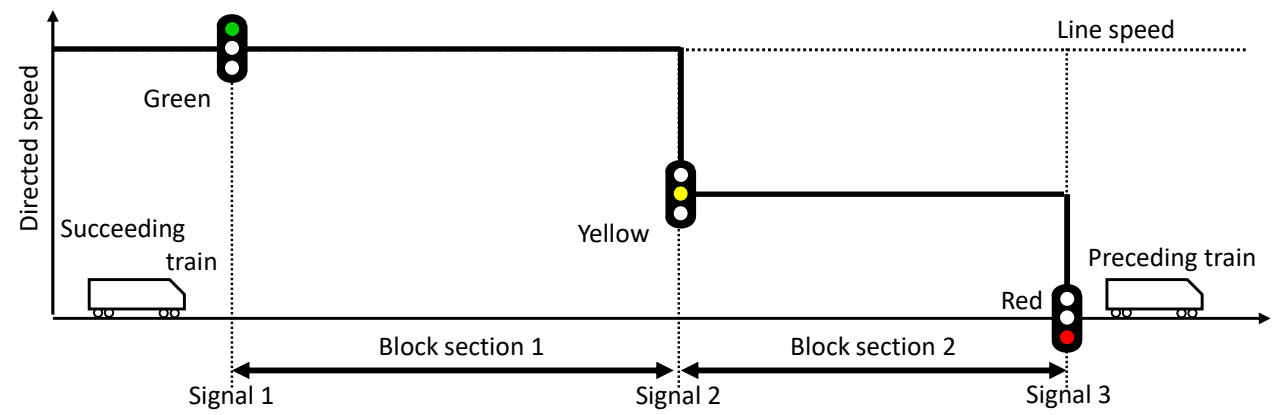

(a)

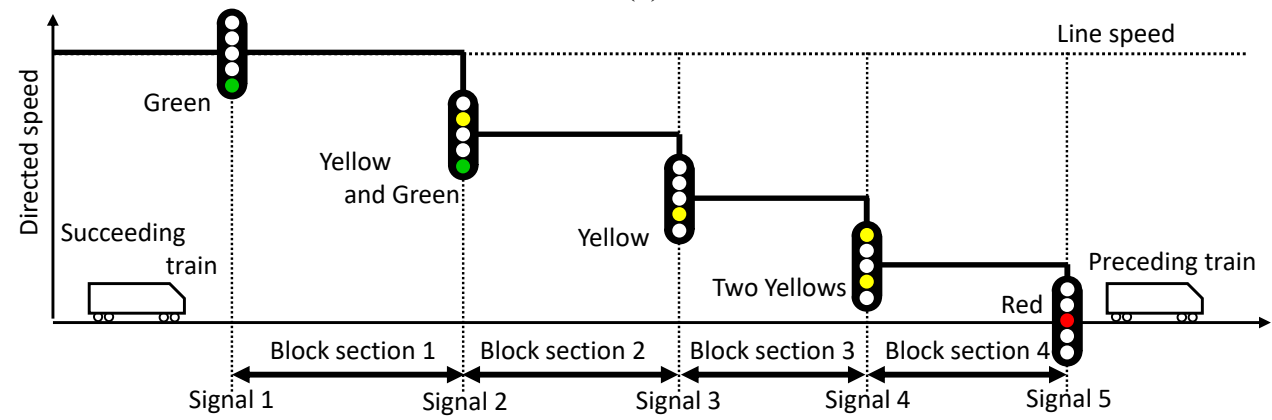

(b)

Figure 2: An example of signal aspect transitions. (a) Signal aspect transitions on general lines; and (b) Signal aspect transitions on high capacity lines.

directed speed (directed speed by a signal) shown in Table 1. The signal aspect of the main signal changes depending on the existence of the preceding train and the signal aspect transitions, and the driver operates the train based on this signal aspect (Fig. 2).

\subsection{Creation of a signal aspect transitions}

The signal aspect transitions shows transitions between each signal aspects [3]. The signal aspect changes based on the signal aspect transition system shown in Fig. 3. The transition between each signal aspect is basically composed of a proceed signal $(\mathrm{G})$, a caution signal $(\mathrm{Y})$, and a stop signal (R) shown by the solid lines in Fig. 3. However, in order to shorten the 


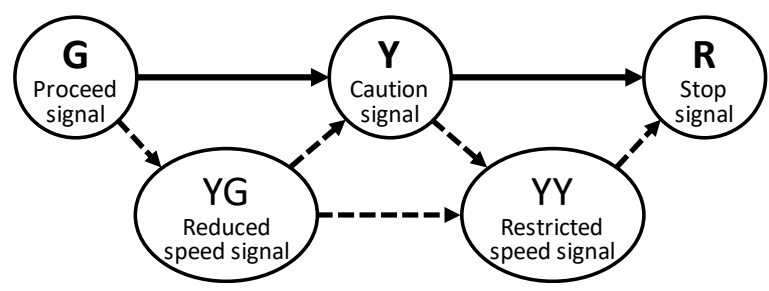

Figure 3: Signal aspect transition system.

headway or to compensate a shortage of deceleration distance, a transition (broken line) of a signal aspect routing through a reduced speed signal (YG) or a restricted speed signal (YY) may be set. In this section, we describe the creation of the signal aspect transitions corresponding to Step 2 of Fig. 1.

\subsubsection{Calculation of running speed at each block section}

In creating the signal aspect transitions, it is necessary to calculate the deceleration distance at each block section (see Section 2.2.2). The deceleration distance can be calculated based on the directed speed set for each signal (see Table 1), with the directed speed as the deceleration initial speed and deceleration final speed. For example, the deceleration distance from $Y$ to $R$ can be calculated as the deceleration distance from $45 \mathrm{~km} / \mathrm{h}$, which is the directed speed of $Y$, to $0 \mathrm{~km} / \mathrm{h}$, which is the directed speed of R. However, since $\mathrm{G}$ is directed to drive within a range not exceeding the line speed, the deceleration initial speed can't be determined. Therefore, in many cases, a deceleration initial speed in the case of $G$ is calculated by a speed profile (a part of a train performance curve) created to examine signal aspect. The deceleration initial speed may be calculated by setting the line speed in the target block section (length of block section) as the deceleration initial speed or by setting the speed at the signal sighting position as the deceleration initial speed. In addition, since the line speed and the stopping station are different depending on the train type, it is necessary to create the speed profile for each train type and to calculate the deceleration initial speed at $G$ based on each profile.

\subsubsection{Calculation of the deceleration distance for each signal aspect transition}

In cases where the train decelerates according to the signal aspect transition setting in each block section, the train must be able to decelerate to the speed directed by the signal aspect within the length of block section or within the signal sighting distance. Therefore, as shown in Section 2.2.1, it is necessary to calculate the deceleration distance corresponding to the signal aspect transition. The visibility of signals is not taken into consideration at the stage of examining the signal arrangement, therefore, the length of the block section is often used as a criterion for evaluation.

Eqn (1) is applied to the calculation of the deceleration distance $S \mathrm{~m}$ for each signal aspect transition. For the gradient of eqn (1), either the minimum gradient value or the average gradient value within each block section is applied. If necessary, the idle running time until the start of deceleration may be added to eqn (1)

$$
S=\frac{V_{0}^{2}-V_{1}^{2}}{7.2 \times\left(\beta+\frac{\theta}{K}\right)},
$$

where 
$V_{0}$ is the deceleration initial speed expressed in $\mathrm{km} / \mathrm{h}$,

$V_{l}$ is the deceleration finial speed expressed in $\mathrm{km} / \mathrm{h}$,

$\beta$ is the braking deceleration rate expressed in $\mathrm{km} / \mathrm{h} / \mathrm{s}$,

$\theta$ is the gradient in $\%$, ${ }^{*}$ Negative value if the gradient is downslope.

$K$ is the inertia coefficient. * 31 for electric multiple-unit trains $/ 30$ for others.

In the examination of the signal arrangement, the braking deceleration rate of eqn (1) and the deceleration initial speed $\left(V_{0}\right)$ for $\mathrm{G}$ are different depending on the train characteristic. For that reason, the deceleration distance is calculated for multiple train types and the maximum value is used. Therefore, in this examination, it is necessary to select multiple train types that may increase the deceleration distance and calculate the maximum value of the deceleration distance.

\subsubsection{Setting of the signal aspect transition}

Based on the length of block and the deceleration distance calculated for each signal transition, the signal transition at each block section is set [3]. For example, as shown in Fig. 4, the deceleration distance from $\mathrm{G}$ to $\mathrm{Y}$ is $520 \mathrm{~m}$ and the deceleration distance from $\mathrm{G}$ to YG is $280 \mathrm{~m}$ in the block section from signal 1 to signal 2. In this case, since the length of block section is $520 \mathrm{~m}$ or more, the signal aspect transition from $\mathrm{G}$ to $\mathrm{Y}$ can be set for signal 1 and signal 2. The signal aspect transition of each signal affects headways. From the viewpoint of headways, it is desirable that the signal aspect of each signal returns to $G$ as soon as possible after the preceding train passed the section. Therefore, considering the signal aspect transition system shown in Fig. 3, it is desirable that the signal aspect transition consists of only G, Y and R, without YG or YY.

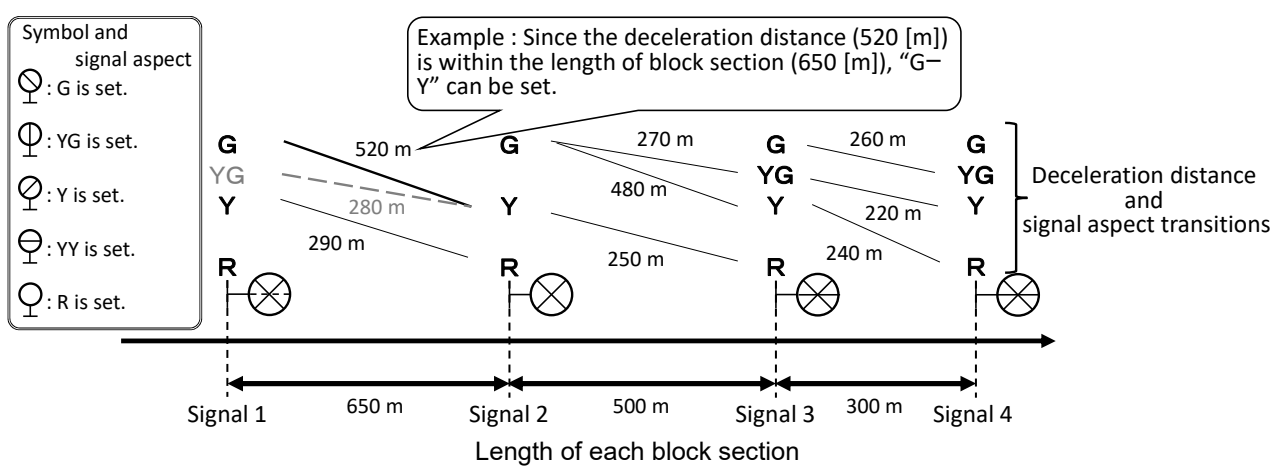

Figure 4: Setting of the signal aspect transition.

\subsection{Calculation of headways}

Two trains running successively must secure a sufficient time interval for safety. This time interval is called the headway [4] and is calculated from the train performance curves of two trains, the signal aspect transitions, and the dwell time. The train performance curve applied at this calculation takes the driving operation into account. Headways are calculated at each signal. As an example, we use Fig. 5 to explain how to calculate the headway at signal 1. The time from when a preceding train passes through signal 1 until when the train passes through the position (signal 3) necessary for signal 1 to show $\mathrm{G}$ again is defined as $T_{l}$. Furthermore, 


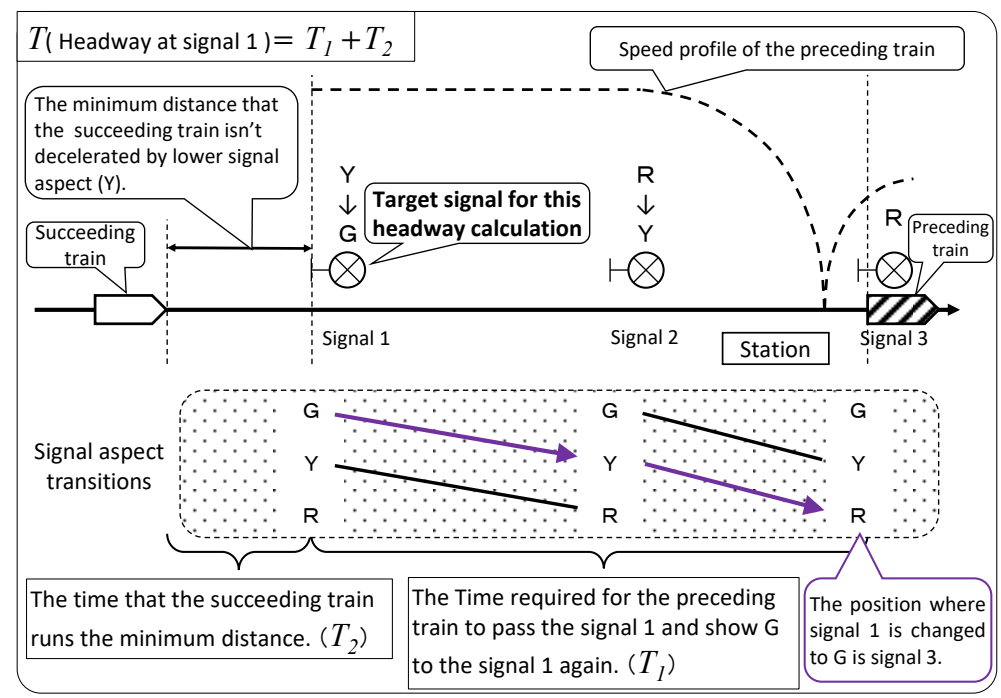

Figure 5: Calculation of a headway [5].

a certain time that a succeeding train runs the minimum distance which the train isn't decelerated by lower signal aspect ( $\mathrm{Y}$ in Fig. 5) is defined as $T_{2}$. Basically, the sum of these $T_{1}$ and $T_{2}$ is the headway $T$ at signal 1 . In some cases, it is necessary for this calculation to consider the delay time caused by the driver's braking operation, the signalling system, and so on.

Headways are expressed by using a headway diagram shown in Fig. 6. The headway diagram is created based on the train movement obtained from train performance curves (time curve) and the signal aspect transitions. The vertical axis of the headway diagram represents position, and the horizontal axis represents time. Furthermore, headways calculated at each signal are displayed on the headway diagram. In the examination of the signal arrangement, the headways at all signals must be smaller than the target headway.

\section{SIGNAL ARRANGEMENT EVALUATION SYSTEM}

The examination of the signal aspect transitions and headways based on the signal arrangement plan (corresponding to Step 2 and Step 3 in Fig. 1) requires a lot of time in the examination of the signal arrangement. In order to support this evaluation (the part surrounded by the red dashed line in Fig. 1), we developed a signal arrangement evaluation system based on the knowledge described in Section 2.

\subsection{Key points of evaluating the signal arrangement}

The results from the investigation shown in Section 2, it was found out that there are two points that must be satisfied in order to decide the signal arrangement.

a) Conditions to be satisfied in the signal aspect transitions

As shown in 2.2, in cases where the train decelerates according to the signal aspect transition setting in each block section, the train is required to decelerate to the speed directed by the signal aspect within the length of the block section. Furthermore, in the calculation of the deceleration distance, it is necessary to select multiple train types that 
may increase the deceleration distance and calculate the maximum value of the deceleration distance.

b) Conditions to be satisfied in headways

Satisfaction of the target headway is very important in the examination of the signal arrangement. Therefore, headways at all signals must be smaller than the target headway.

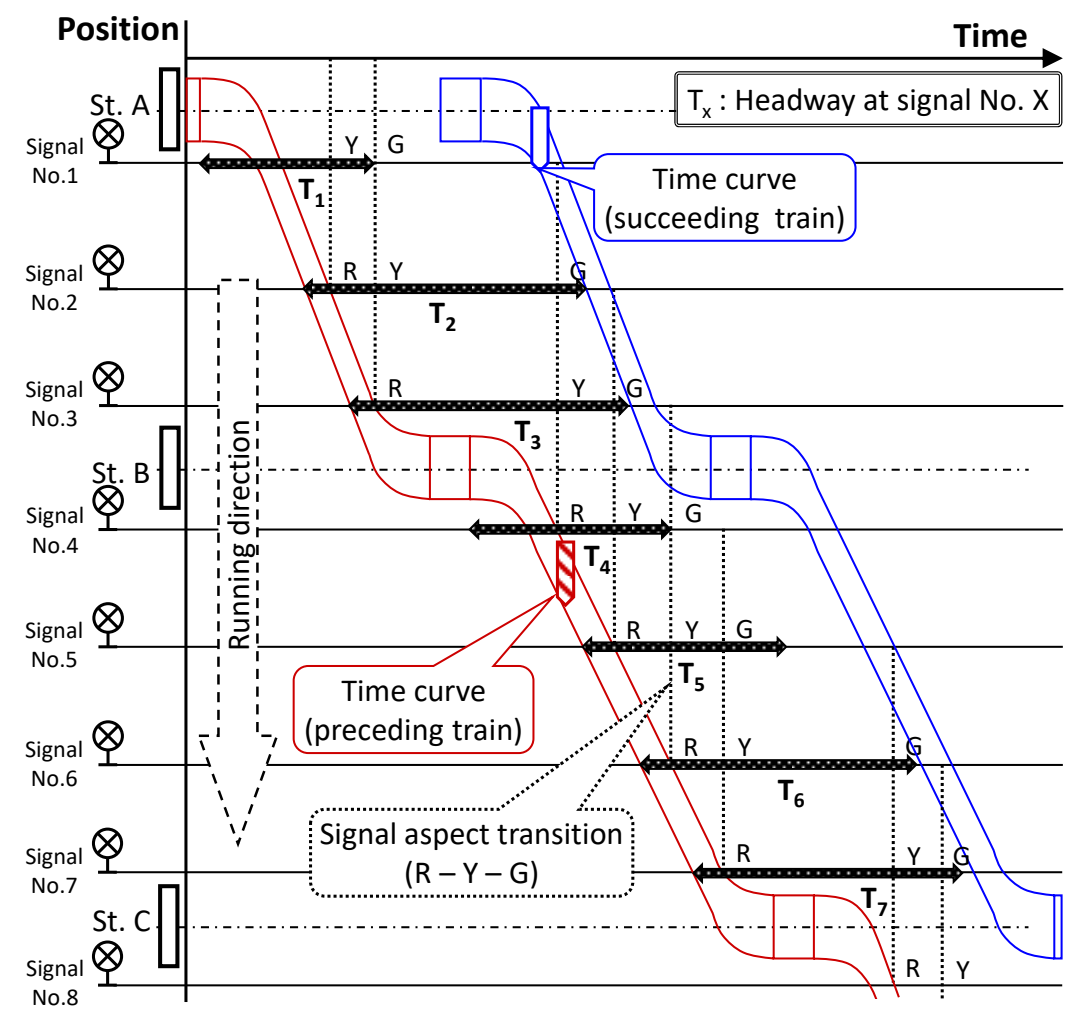

Figure 6: Headway diagram.

In the examination of the signal arrangement, after satisfying the above conditions, the signal arrangement is determined in consideration of the number of traffic lights, the margin for the target headway, and so on. Therefore, the signal arrangement evaluation system to be developed is required to evaluate the signal aspect transitions and the headway. The functions corresponding to each evaluation are summarized below.

a) Evaluation function of the signal aspect transitions

- Display the signal aspect transition.

- Calculate deceleration distance for each signal aspect transition.

- Create speed profiles for multiple train types.

- Calculate the deceleration distance for multiple vehicle types and output the maximum value.

- Display the length of block section. 
- Confirm whether the deceleration distance for each signal aspect transition is no more than the length of each block section or not.

b) Evaluation function of the headway

- Calculate headways at each signal.

- Confirm that headways at each signal satisfy the target headway.

\subsection{Development of the signal arrangement evaluation system}

As shown in Section 3.1, the signal arrangement evaluation system requires the function of evaluating the signal aspect transitions and the function of evaluating the headway. Therefore, as shown in Fig. 7, we developed the system that integrates the evaluation function of the signal aspect transition and the headway calculation function.

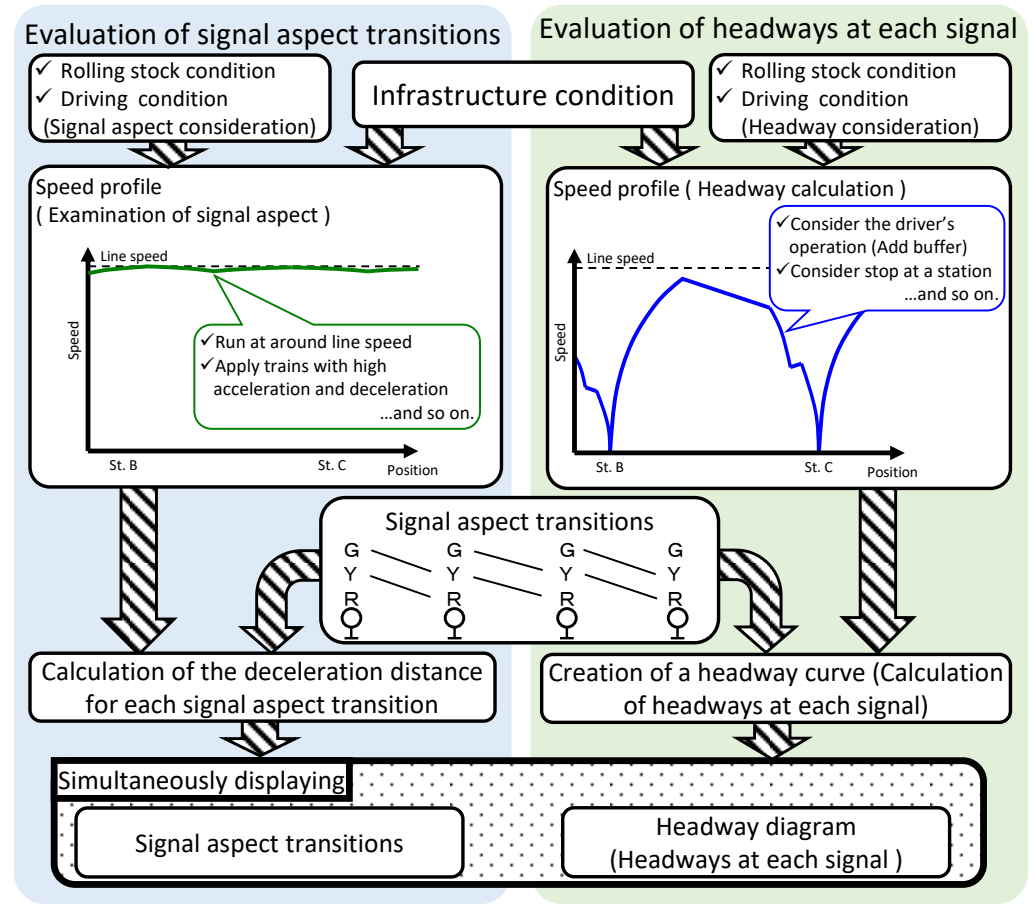

Figure 7: Configuration of the signal arrangement evaluation system.

We have developed a running time and headway calculation system [6], [7] that has a function of calculating headways. The function can calculate headways at each signal. Furthermore, this function has equivalence to the evaluation function of the headway shown in Section 3.1 because headways at each signal can be displayed on the headway diagram. Therefore, we decided to develop the signal arrangement evaluation system based on the system. On the other hand, we developed new functions for the evaluation of the signal aspect transition based on the contents summarized in Section 3.1. 
3.2.1 Creation of a speed profile for examination of signal aspects

In order to evaluate the signal aspect transitions, it is necessary to calculate running speed at each block section shown in Section 2.2.1. In order to calculate running speed, the speed profile for examination of signal aspects is required. As a result of the investigation in Section 2 , it is confirmed that this speed profile is different from that applied when calculating headways. The conditions for creating each speed curve are summarized as follows.

a) Speed profile for examination of signal aspects

In the evaluation of the signal aspect transitions, the speed profile is required, which is created under the condition that the deceleration distance is maximum. The following conditions must be satisfied to create the speed profile that maximizes the deceleration distance.

- The buffer time caused by the driving operation is not considered.

- At each station, trains mainly pass through.

- Applied trains are selected in consideration of the following.

$\circ$ Trains with low deceleration rate and long idling time before braking is activated

ex) Freight trains and electric multiple-unit trains driven by locomotives

$\circ \quad$ Trains with high deceleration initial speed

ex) Trains mainly pass and have a high maximum speed or trains with a high acceleration performance

b) Speed profile for the headway calculation

In the headway calculation, the speed profile used for creating the transport planning is applied. The creation conditions are as shown below.

- The buffer time caused by the driving operation is considered.

- Stops and passages at each station are set based on the transport planning.

- Trains with low rolling stock performance are applied to avoid running time shortage.

\subsubsection{Calculation of the deceleration distance}

In order to evaluate the signal aspect transitions, it is necessary to calculate the deceleration distance corresponding to a signal aspect transition setting in each block section based on the speed profile for examination of signal aspects. The method of calculating the deceleration distance $S \mathrm{~m}$ is as shown in Section 2.2.2, but we considered the following points as more realistic conditions.

- Considering idling time until braking is activated, the eqn (2) including idling time is applied to the deceleration distance calculation. The average gradient is used as the gradient value applied to the eqn (2)

$$
S=\frac{V_{0}^{2}-V_{1}^{2}}{7.2 \times(\beta+\theta / K)}+\frac{V_{0}}{3.6} \times t
$$

where

$V_{0}$ is the initial deceleration speed expressed in $\mathrm{km} / \mathrm{h}$,

$V_{l}$ is the final deceleration speed expressed in $\mathrm{km} / \mathrm{h}$,

$B$ is the braking deceleration rate expressed in $\mathrm{km} / \mathrm{h} / \mathrm{s}$,

$\theta$ is the gradient expressed in \%o, * Negative value if the gradient is downslope. 
$K$ is the inertia coefficient $* 31$ for electric multiple-unit trains/ 30 for others $t$ is idling time expressed in second.

- It is necessary to consider the deceleration distance of trains with different train performance. Therefore, it enables the calculation of two speed profiles for examination of signal aspects under different train conditions.

\subsubsection{Display of signal aspect transitions and headway diagram}

In the examination of the signal arrangement, it is necessary for the person in charge to confirm the signal aspect transitions and the headway diagram at the same time. Therefore, it is necessary for the system to be developed to simultaneously display the signal aspect transitions and the headway diagram. As described above, this system utilizes the function of calculating headways of the running time and headway calculation system. Therefore, in this system, it is possible for the signal aspect transitions that is the basis of the headway calculation to be displayed together with the headway diagram. Furthermore, the length of block sections and the deceleration distance for each signal aspect transition are displayed based on the deceleration distance calculation results shown in Section 3.2.2. If the deceleration distance is longer than the length of block section, the difference is displayed as the deceleration distance shortage. As a result, it is possible for the person in charge to confirm the satisfaction of the signal aspect transitions and the headways at all signals, which are indispensable for the examination of the signal arrangement.

\section{EVALUATION OF THE DEVELOPED SYSTEM}

In order to evaluate the developed system, we will carry out an examination of the signal arrangement on the model line.

\subsection{Overview of the model line}

The line conditions that require a lot of time for the examination of the signal arrangement are lines with short headway and lines where run electric multiple-unit trains and freight trains with different deceleration distances. Considering these conditions, the line shown in Fig. 8 is used as the model line.

- Ground equipment conditions: The line length is approximately $15 \mathrm{~km}$, and there are 20 signals. There are many downward slopes toward the end point.

- Headway calculation conditions: Headways at all signals from station E to station A is calculated based on the train performance curve shown in Fig. 8. In addition, the target headway for this section is assumed to be 3 minutes.

- Evaluation conditions of the signal aspect transitions: The deceleration distance is calculated for the following two types with different train performance.

$\circ \quad$ Train type 1: This train stops at station $\mathrm{E}$ and station A. This train is a freight train with a maximum speed of $75 \mathrm{~km} / \mathrm{h}$, the braking deceleration rate of $1.7 \mathrm{~km} / \mathrm{h} / \mathrm{s}$, and idling time of 8 seconds.

- Train type 2: This train stops at station E and station A stop. This train is an electric multiple-unit train with a maximum speed of $100 \mathrm{~km} / \mathrm{h}$, the braking deceleration rate of $2.5 \mathrm{~km} / \mathrm{h} / \mathrm{s}$, and idling time of a second. 


\subsection{The results of evaluation}

As shown in Fig. 9, we confirmed that the satisfaction of the signal aspect transitions and headways at each signal, which are indispensable for the examination of the signal arrangement, can be evaluated at the same time. Moreover, the time required to calculate the evaluation result is several seconds.

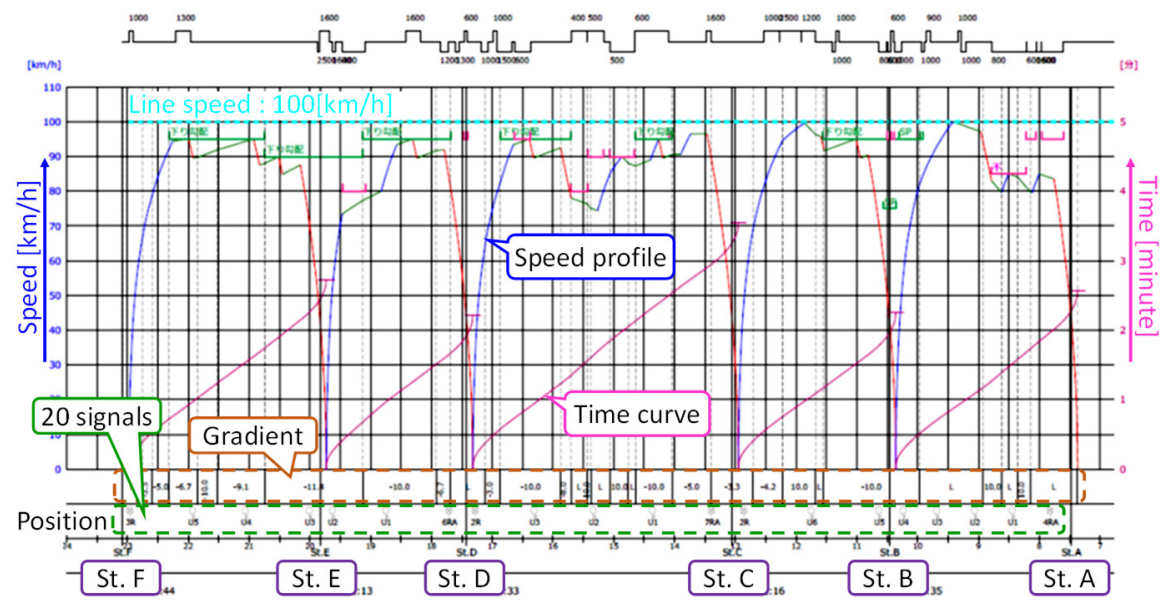

Figure 8: Train performance curve of the model line.

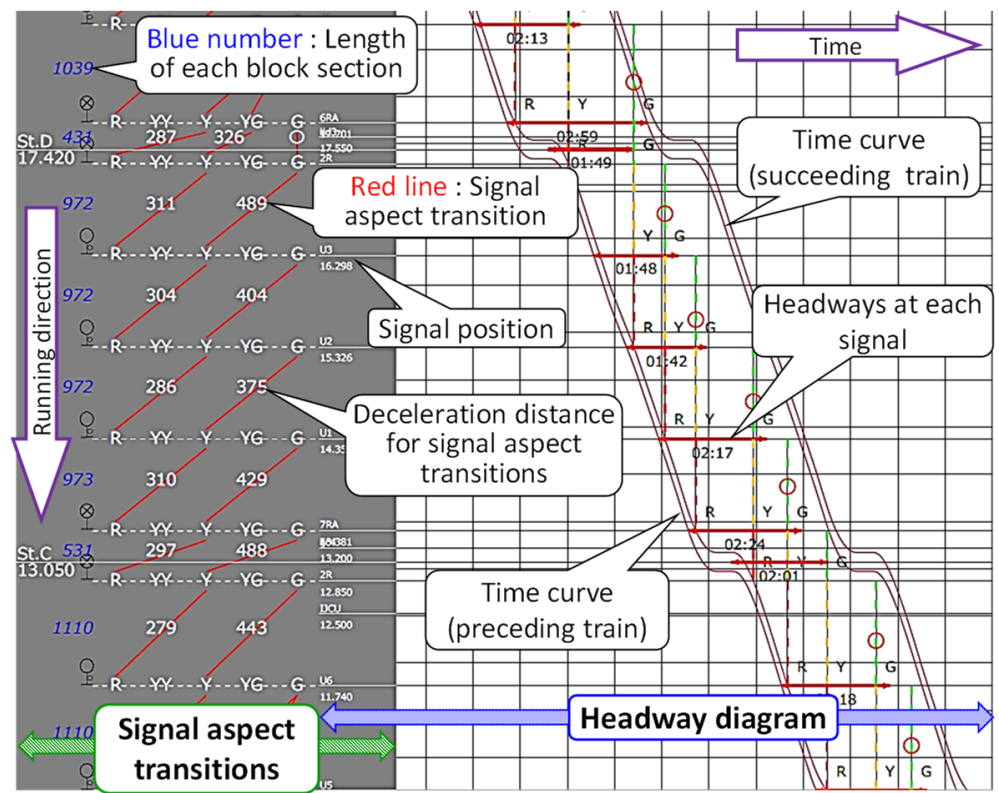

Figure 9: Evaluation results from the developed system. 
a) The evaluation result of conditions to be satisfied in the signal aspect transitions From the signal aspect transitions display section of Fig. 8, whether the deceleration distance is sufficient can be confirmed by displaying the length of block sections and the deceleration distance for each signal aspect transition. For the deceleration distance, the larger value of the deceleration distance calculation results of two train models is applied.

b) The evaluation result of conditions to be satisfied in headways

Satisfaction of the target operation time interval can be confirmed from headways at all signals displayed on the headway diagram.

It takes a lot of time for the person in charge to perform the same work without developed system. This system is considered effective in supporting the examination of the signal arrangement, because the evaluation result of a signal arrangement can be obtained within a few seconds.

\section{CONCLUSION AND FUTURE WORKS}

When planning new line construction and track layouts improvement, it is necessary to determine the position of each signal, called an examination of the signal arrangement, in consideration of train operation and construction costs. The examination of the signal arrangement requires a lot of time and a person who has the knowledge of signalling system and train operation. For that reason, system assistance is required to carry out the examination of the signal arrangement.

Therefore, in order to solve these problems, we have developed a signal arrangement evaluation system. First, we have conducted hearing investigations with the person in charge of the examination of the signal arrangement to understand the overview of its. Based on the knowledge we have obtained from these investigations, we have developed the system that can evaluate the signal transitions and headways at each signal. In addition, using the developed system, we have evaluated a signal arrangement of a model line, which requires consideration of two train types with different deceleration performance and has 20 signals. As a result, we have confirmed that it is possible to evaluate the signal arrangement in a few seconds (Fig. 9). We conclude that the developed system can shorten the time required for the examination of the signal arrangement.

The developed system introduced in this paper is planned to be developed further for practical use. In addition, as future works, we plan to construct a method that proposes a signal arrangement based on the target headway, instead of evaluating the signal arrangement.

\section{REFERENCES}

[1] Theeg, G. \& Vlasenko, S. (eds), Railway Signalling and Interlocking, 2nd ed., PMC Media House: Hamburg, 2018.

[2] Ugajin, H., Shiroto, H., Fujinami, K. \& Omino, K., Two greens: A new signal aspect for high speed train on wayside signaling. Quarterly Report of RTRI, 40(1), pp. 13-17, 1999.

[3] Yoshitake, I., Tetsudo no Untenhoan (Train Protection Facilities on Railway), Japan Train Operation Association: Tokyo, 2006. (In Japanese.)

[4] Kuramoto, K., Kotani, T., Hirano J. \& Akatsu N., Untenriron - Saikaiteiban (Theory for Efficient Train Operation - Revised Edition), Japan Train Operation Association: Tokyo, 2010. (In Japanese.)

[5] Kumazawa, K. \& Takeuchi, Y., Construction of a comparative evaluation method of the driving operation form multiple viewpoints. RTRI Report, 31(10), pp. 47-52, 2017. (In Japanese.) 
[6] Hirano, J., Tomii, N., Yamashita, O. \& Kimura, Y., Development a train performance computation system on an engineering workstation. Computer in Railway III, pp. 127136, 1992.

[7] Railway Technical Research Institute, Signalling and Transport Information Technology Division, Running time and headway calculation system - SPEEDY. www.rtri.or.jp/rd/division/rd47/rd4740/rd47400101.html. Accessed on: 17 Apr. 2020. (In Japanese.) 